\title{
Clinical significance of reduced GPRC5A expression in surgically resected non-small cell lung cancer
}

\author{
ER JIN $^{1^{*}}$, WENZHE WANG ${ }^{2 *}$, MENGDIE FANG $^{2 *}$, WEI WANG $^{3}$, RUIFEI XIE ${ }^{4}$, \\ HONG ZHOU ${ }^{3}$, JIAN YE ${ }^{1}$, RUJUN XU ${ }^{3}$ and SHENGLIN MA ${ }^{4}$ \\ ${ }^{1}$ Department of Respiratory Medicine, Nanjing Medical University, Affiliated to Hangzhou First People's Hospital, \\ Hangzhou, Zhejiang 310006; ${ }^{2}$ Center for Molecular Medicine, Zhejiang Academy of Medical Sciences, \\ Hangzhou, Zhejiang 310012; Departments of ${ }^{3}$ Pathology and ${ }^{4}$ Medical Oncology, Nanjing Medical University, \\ Affiliated to Hangzhou First People's Hospital, Hangzhou, Zhejiang 310006, P.R. China
}

Received December 8, 2017; Accepted September 17, 2018

DOI: $10.3892 / \mathrm{ol} .2018 .9537$

\begin{abstract}
G protein-coupled receptor, family C, group 5 member A (GPRC5A) is a retinoid-inducible protein, which has been characterized as a tumor-suppressor gene in lung cancer. The present study further examined GPRC5A expression in non-small cell lung cancer (NSCLC) for any association with the clinical features and treatment outcomes of patients with NSCLC. A total of 30 paired NSCLC tumor and adjacent normal tissues were analyzed for the detection of GPRC5A mRNA and protein using reverse transcription-quantitative polymerase chain reaction (RT-qPCR) and western blot analysis, respectively. Immunohistochemistry was performed to determine the GPRC5A expression levels in 110 NSCLC and 60 para-tumor tissues. The results confirmed significantly lower expression levels of GPRC5A in NSCLC tumors compared with the corresponding noncancerous tissues $(\mathrm{P}<0.001)$. Lost GPRC5A expression was significantly associated with the tumor histological type $(\mathrm{P}=0.008)$, poor tumor differentiation $(\mathrm{P}<0.001)$ and tumor-node-metastasis (TNM) stage $(\mathrm{P}<0.001)$. Kaplan-Meier curve analysis revealed that patients with NSCLC with low GPRC5A expression tumors had a worse prognosis compared with those with high GPRC5A expression tumors $(\mathrm{P}=0.010)$. The results of
\end{abstract}

Correspondence to: Dr Shenglin Ma, Department of Medical Oncology, Nanjing Medical University, Affiliated to Hangzhou First People's Hospital, 261 Huansha Road, Hangzhou, Zhejiang 310006, P.R. China

E-mail: amyier@163.com

Dr Rujun Xu, Department of Pathology, Nanjing Medical University, Affiliated to Hangzhou First People's Hospital, 261 Huansha Road, Hangzhou, Zhejiang 310006, P.R. China

E-mail: xurj@hotmail.com

*Contributed equally

Key words: GPRC5A, non-small cell lung carcinoma, immunohistochemistry, prognosis multivariate Cox analysis further suggested that low GPRC5A expression was an independent prognostic factor for patients with NSCLC $(\mathrm{P}<0.001)$. The results of this study suggest GPRC5A expression has clinical potential as a prognostic biomarker for patients with NSCLC.

\section{Introduction}

Lung cancer is the most common cancer and the leading cause of cancer-related mortality in the world (1). In China, lung cancer has become the number one health issue as the incidence and mortality rates have dramatically increased in the past decades (2). Histologically, lung cancer can be divided into two types, i.e., small cell lung cancer and non-small cell lung cancer (NSCLC). Approximately $85 \%$ of lung cancer cases are NSCLC, and the majority of these patients are diagnosed at late stages (1). Despite the tremendous efforts and progress made in lung cancer research, such as the use of aggressive multimodal chemo-, radio-, and targeted therapy, the overall treatment outcome for NSCLC patients still remains poor. The optimal treatment of lung cancer relies on accurate disease staging and potential prediction of treatment outcomes; thus, there is an urgent need for the identification and evaluation of novel early detection methods and prognostic markers for NSCLC.

$\mathrm{G}$ protein-coupled receptor, family $\mathrm{C}$, group 5 member $\mathrm{A}$ (GPRC5A) was originally identified as an all-trans-retinoic acid-inducible protein $(3,4)$, which consists of an extracellular ligand-binding domain and an internal transmembrane domain with seven membrane-spanning $\alpha$-helices and an internal C-terminal domain (5). The expression of GPRC5A is low in the liver, pancreas, colon, and mammary glands; but it is highly expressed in normal lung tissue. Thus, it is considered as a lung cancer suppressor gene $(3,5-7)$. Previous studies have provided useful insights into the role of GPRC5A in lung cancer development and progression. For example, Tao et al have reported that in GPRC5A-/- mice, 17\% developed lung adenocarcinoma and $76 \%$ developed lung adenoma (8). Exposure of GPRC5A-knockout mice to the carcinogen nicotine-derived nitrosamine ketone found in tobacco or infection with Haemophilus also has been reported to cause 
the development of lung adenoma and adenocarcinoma $(9,10)$. In addition, deletion of GPRC5A leads to enhancement of the transformed phenotype of normal and malignant lung epithelial cells via Stat3 and downstream signaling activation (3). A very recent study further revealed a potential role of GPRC5A in inhibition of epidermal growth factor receptor expression and activation (11). Together, these studies indicate the potential of GPRC5A as an antitumor target for human lung cancer patients. Thus, the aim of this study was to assess the expression of GPRC5A mRNA and protein as well as to investigate the correlations of GPRC5A expression with the clinicopathological features and survival rates of NSCLC patients.

\section{Materials and methods}

Patients and tissue samples. A total of 110 NSCLC patients, who had a surgical tumor resection at Nanjing Medical University Affiliated Hangzhou Hospital (Hangzhou, China) between March 2007 and December 2009, were retrospectively recruited for immunohistochemical analysis of GPRC5A in their tissue specimens. Among these specimens, there were 64 cases of lung adenocarcinoma and 46 cases of squamous cell carcinoma. Moreover, 30 paired fresh snap-frozen NSCLC and adjacent noncancerous lung tissues were also used for reverse transcription-quantitative polymerase chain reaction (RT-qPCR) and western blot analysis of GPRC5A. None of the patients included in this study received chemotherapy or radiotherapy prior to surgery. The clinicopathological data of the patients were obtained from medical records. Postoperative pathological staging was determined according to the seventh edition of the tumor-node-metastasis (TNM) classification system (12). The inclusion criteria of the patients in this study were as follows: completed surgical tumor resection, the patient survived for more than three months after surgery, and the cause of death within the five years following surgery was not due to any cause other than lung cancer. Patient follow-up was performed via a phone interview, and the median follow-up time was 38 months (ranging between 8 and 71 months). The last follow-up date was December 2014. Informed consents were obtained from all patients before operation, and all the procedures were approved by the Medical Ethics Committee of the Affiliated Hangzhou Hospital of Nanjing Medical University. The patient characteristics are summarized in Table I.

$R T-q P C R$. Total RNA was isolated from tissues using TRIzol (Invitrogen; Thermo Fisher Scientific, Inc., Waltham, MA, USA) and reverse transcribed into cDNA using the Thermoscript RT System (Invitrogen; Thermo Fisher Scientific, Inc.), according to the manufacturer's instructions. qPCR was performed using the cDNA and an ABI7000 sequence detector (Applied Biosystems; Thermo Fisher Scientific, Inc.) with SYBR-Green PCR Master Mix (Applied Biosystems; Thermo Fisher Scientific, Inc.), according to the manufacturer's protocol. GAPDH was used as an internal control, and the relative level of GPC5A mRNA expression was analyzed using the $2^{-\Delta \Delta \mathrm{Cq}}$ method (13). The following primers were used: GPRC5A, 5'-GCCTCACCTTCGCCT TCATC-3' and 5'-CAACTCGTTTCGATTTCTGACAA-3'; GAPDH, 5'-GGCTGAGAACGGGAAGCTTGTCAT-3' and
5'-CAGCCTTCTCCATGGTGGTGAAGA-3'; and negative control, 5'-UUCUCCGAACGUGUCACGUTT-3'.

Protein extraction and western blot. Total cellular protein was extracted from frozen tissue specimens using a tissue grinder and RIPA lysis buffer. Equal amounts of protein sample $(20 \mu \mathrm{g})$ were subjected to sodium dodecyl sulfate-polyacrylamide gel electrophoresis and transferred onto a nitrocellulose membrane (EMD Millipore, Billerica, MA, USA). Membranes were incubated with a primary rabbit polyclonal anti-GPRC5A antibody (1:100; Proteintech, Wuhan, China). Anti-GAPDH antibody was used as the loading control (Cell Signaling Technology, Inc., Danvers, MA, USA). Positive protein bands were visualized by using the Enhanced Chemiluminescence Detection System (EMD Millipore), and the optical density of target bands was quantified using Imager software (Alpha Corporation, San Leandro, CA, USA).

Immunohistochemistry. Surgically resected tissue specimens were fixed in $10 \%$ neutral formalin, embedded in paraffin, and cut into $4-\mu \mathrm{m}$-thick sections. Immunostaining was performed using a standard avidin-biotin-peroxidase complex method. Sections were deparaffinized in xylene and rehydrated in graded alcohol solutions. Antigen retrieval was performed by boiling the sections in $0.01 \mathrm{M}$ citrate buffer for $2 \mathrm{~min}$ in an autoclave. Hydrogen peroxide was used to block endogenous peroxide activity, and normal goat serum was used to reduce nonspecific binding. After preparation, the sections were incubated at room temperature for $2 \mathrm{~h}$ with the polyclonal anti-GPRC5A antibody (1:100). Mouse immunoglobulin was used as a negative control. Biotinylated goat anti-mouse IgG was used as the secondary antibody. After incubation with the secondary antibody, the sections were washed and incubated with streptavidin-biotin conjugated with horseradish peroxidase. The peroxidase reaction was developed in a 3,3'-diaminobenzidine tetrahydrochloride solution.

Evaluation and scoring of immunostained sections. Two pathologists independently reviewed and scored each stained tissue section, and five fields with at least 100 cells in each field were evaluated per section. Data were assessed and scored according to the staining percentage and intensity. The positive staining percentage was scored as follows: $0(0-9 \%)$, $1(10-25 \%), 2(26-50 \%), 3(51-75 \%)$, or $4(76-100 \%)$. The intensity was scored as follows: 0 (negative staining), 1 (weak staining), 2 (moderate staining), or 3 (strong staining). The combined total score was the product of these two numbers, ranging from 0 to 12 . The cutoff scores for high and low expression were determined based on the heterogeneity value measured by the log-rank test with respect to overall survival. A staining index score $\geq 6$ defined tumors with high GPRC5A expression, and a score $<6$ indicated low GPRC5A expression.

Statistical analysis. SPSS 17.0 software (SPSS, Inc., Chicago, IL, USA) was used to perform all statistical analyses. A value of $\mathrm{P} \leq 0.05$ was considered statistically significant. The expression level of GPRC5A in cancer tissues was further divided into high or low categories according to the median immunostaining score. The $\chi^{2}$ test was used to analyze the associations of the GPRC5A expression score with various 
Table I. GPRC5A protein expression and non-small cell lung cancer clinicopathological features.

\begin{tabular}{|c|c|c|c|c|}
\hline Clinicopatholigical features & Cases & GPRC5A low & GPRC5A high & P-value \\
\hline \multicolumn{5}{|l|}{ Sex } \\
\hline Male & 64 & 43 & 21 & \multirow[t]{2}{*}{0.829} \\
\hline Female & 46 & 30 & 16 & \\
\hline \multicolumn{5}{|l|}{ Age (years) } \\
\hline$<61$ & 54 & 33 & 21 & \multirow[t]{2}{*}{0.252} \\
\hline$\geq 61$ & 56 & 40 & 16 & \\
\hline \multicolumn{5}{|l|}{ Tumor size (cm) } \\
\hline$\leq 3$ & 59 & 39 & 20 & \multirow[t]{2}{*}{0.95} \\
\hline$>3$ & 51 & 34 & 17 & \\
\hline \multicolumn{5}{|l|}{ Smoking } \\
\hline Nonsmoker & 28 & 19 & 9 & \multirow[t]{2}{*}{0.846} \\
\hline Smoker & 82 & 54 & 28 & \\
\hline \multicolumn{5}{|l|}{ Histological type } \\
\hline $\mathrm{SCC}$ & 46 & 37 & 9 & \multirow[t]{2}{*}{0.008} \\
\hline Adenocarcinoma & 64 & 36 & 28 & \\
\hline \multicolumn{5}{|l|}{ Tumor differentiation } \\
\hline Well-moderate & 56 & 51 & 5 & \multirow[t]{2}{*}{$<0.001$} \\
\hline Poor & 54 & 22 & 32 & \\
\hline \multicolumn{5}{|l|}{ TNM stage } \\
\hline $\mathrm{I}$ & 69 & 63 & 6 & \multirow[t]{2}{*}{$<0.001$} \\
\hline II-III & 41 & 10 & 31 & \\
\hline
\end{tabular}

TNM, tumor node metastasis; G protein-coupled receptor, family C, group 5 member A; SCC, squamous cell carcinoma.

clinicopathological parameters. The Kappa test was performed to determine the significance of GPRC5A expression in NSCLC and nontumorous tissues. A Kaplan-Meier survival curve was created and compared with the log-rank test results. The Cox proportional hazards regression model was used for univariate and multivariate analyses to explore the effects of the clinicopathological variables and GPRC5A expression on survival.

\section{Results}

Reduced GPRC5A mRNA and protein expression in NSCLC tissues. We first examined GPRC5A expression in 30 paired specimens of NSCLC and adjacent noncancerous tissues and found that the mRNA levels of GPRC5A were significantly lower in NSCLC tissues than in the adjacent noncancerous tissues (mean \pm SEM: $0.24 \pm 0.09$ in tumor tissues vs. $0.60 \pm 0.17$ in adjacent noncancerous tissues, $\mathrm{P}<0.05$; Fig. 1A). Western blot analysis detected a lower level of GPRC5A protein in tumor tissues compared with adjacent noncancerous tissues (mean \pm SEM: 0.66 \pm 0.22 in tumor tissues vs. $1.59 \pm 0.36$ in adjacent noncancerous tissues, $\mathrm{P}<0.05$; Fig. $1 \mathrm{~B}$ and $\mathrm{C}$ ).

Lost expression of GPRC5A protein in NSCLC tissues. Next, we performed immunohistochemical staining of GPRC5A protein in 110 NSCLC and 60 nontumorous tissue samples (Fig. 2). Of these 110 NSCLC specimens, 68 specimens (61.81\%) had lower
GPRC5A protein expression $(\mathrm{P}<0.001)$ compared with that of the normal lung tissues. Specifically, the immunostaining scores of GPRC5A expression were between 0 and 12 in 64 cases of lung adenocarcinomas, with 17 (26.5\%) cases showing no GPRC5A expression (score of 0) and 36 (56.25\%) cases showing weak GPRC5A expression in tumor cells. In 46 cases of lung squamous cell carcinoma, the immunostaining scores of GPRC5A expression were between 0 and 8, with 23 cases showing weak GPRC5A expression and 23 cases showing no GPRC5A expression (score 0). In the lung adenocarcinoma and adjacent noncancerous lung tissues, we noticed that GPRC5A staining was predominantly presented in the cytoplasm and that there was no nuclear staining of GPRC5A protein. However, we observed nuclear staining of GPRC5A protein in 19 of 46 cases $(41.3 \%)$ of lung squamous cell carcinoma.

Reduced GPRC5A expression and the overall survival of NSCLC patients. The 5-year survival rate of the 110 NSCLC patients enrolled in this study was $41.8 \%$. Kaplan-Meier survival curves indicated that reduced GPRC5A expression was associated with a shorter survival time when compared to patients with GPRC5A-expressing tumors (5-year survival rates of 26.3 and $79.2 \%$, respectively; log-rank test, $\chi^{2}=12.57$, $\mathrm{P}<0.001$; Fig. 3). Cox's univariate and multivariate hazard regression model analyses revealed that low expression of GPRC5A was significantly associated with poor tumor differentiation $(\mathrm{P}<0.001)$, the histological type $(\mathrm{P}=0.008)$, and the TNM stage $(\mathrm{P}<0.001)$ of the NSCLC patients. However, 

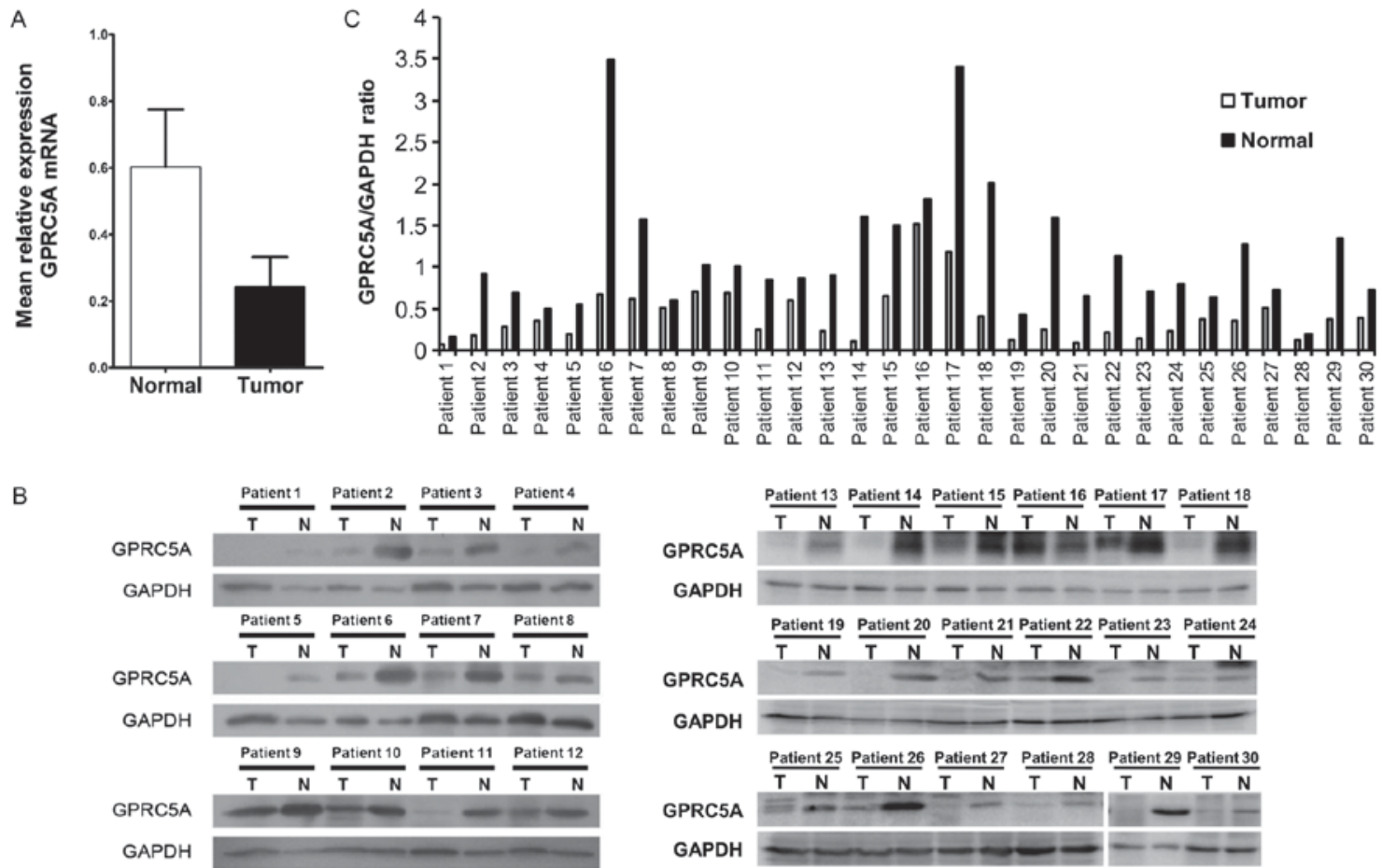

Figure 1. GPRC5A expression in paired NSCLC and adjacent noncancerous tissues. (A) Significance of GPRC5A mRNA expression in NSCLC and paired adjacent nontumorous tissues determined by quantitative reverse transcription-polymerase chain reaction. (B) Western blot analysis of GPRC5A protein expression in paired NSCLC tissues. (C) GPRC5A protein expression quantitated from the western blot results (B). The data represent the average of three independent experiments. Error bars indicate standard deviation. NSCLC, non-small cell lung cancer; GPRC5A, G protein-coupled receptor, family C, group 5 member A.

\section{A}

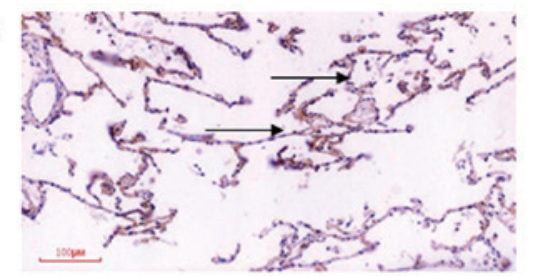

C

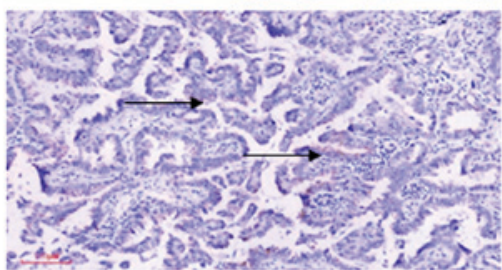

E

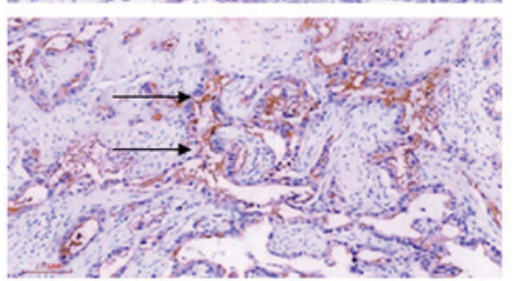

G

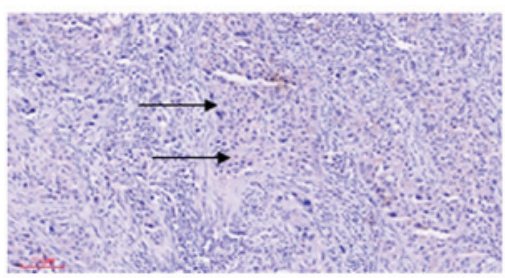

B

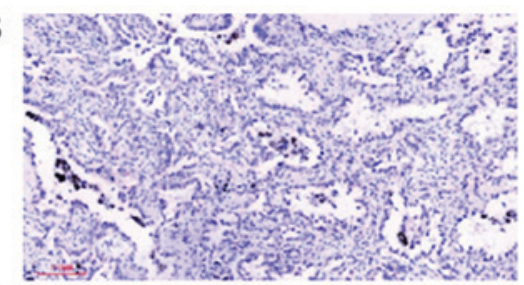

D

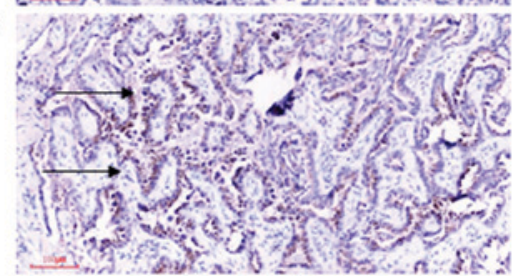

$\mathbf{F}$

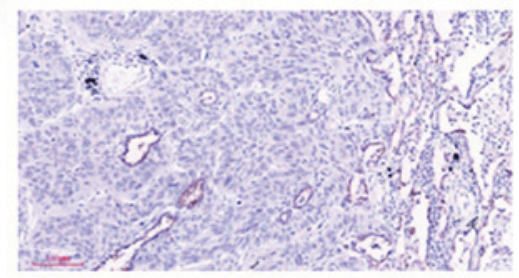

$\mathbf{H}$

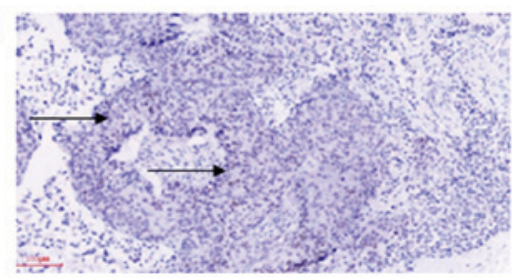

Figure 2. Immunohistochemical staining of GPRC5A protein in non-small cell lung cancer specimens. (A) Staining of GPRC5A in normal tissues adjacent to the adenocarcinoma. (B) Negative staining of GPRC5A in adenocarcinoma tissues. (C) Weak staining of GPRC5A in adenocarcinoma tissues. (D) Moderate staining of GPRC5A in adenocarcinoma tissues. (E) Strong staining of GPRC5A in adenocarcinoma tissues. (F) Negative staining of GPRC5A in SCC tissues. (G) Weak staining of GPRC5A in SCC tissues. (H) Moderate staining of GPRC5A in SCC tissues. Arrows show the positive staining. magnification, x200. SCC, squamous cell carcinoma; GPRC5A, G protein-coupled receptor, family C, group 5 member A. 
Table II. Univariate and multivariate analysis of prognostic factors in 110 NSCLC patients.

\begin{tabular}{|c|c|c|c|c|c|c|}
\hline \multirow[b]{2}{*}{ Characteristics } & \multicolumn{3}{|c|}{ Univariate analysis } & \multicolumn{3}{|c|}{ Multivariate analysis } \\
\hline & P-value & HR & $95 \% \mathrm{CI}$ & P-value & HR & $95 \% \mathrm{CI}$ \\
\hline Sex (male vs. female) & 0.151 & 1.625 & $0.881-3.151$ & 0.460 & 1.308 & $0.642-2.668$ \\
\hline Age ( $<61$ vs. $\geq 61$ years) & 0.197 & 0.981 & $0.952-1.010$ & 0.043 & 0.967 & $0.936-0.998$ \\
\hline Histology (SCC vs. adenocarcinoma) & 0.060 & 0.548 & $0.293-1.026$ & 0.011 & 0.381 & $0.181-0.802$ \\
\hline Differentiation (poor vs. well-moderate) & 0.028 & 0.468 & $0.238-0.921$ & 0.686 & 1.183 & $0.524-2.670$ \\
\hline Tumor size ( $\leq 3$ vs. $>3 \mathrm{~cm})$ & 0.754 & 0.901 & $0.469-1.731$ & 0.035 & 2.249 & $1.058-4.779$ \\
\hline TNM (I vs. II-III) & 0.021 & 0.359 & $0.150-0.855$ & 0.852 & 0.906 & $0.320-2.561$ \\
\hline GPRC5A expression (low vs. high) & 0.001 & 0.215 & $0.084-0.548$ & 0.001 & 0.104 & $0.28-0.384$ \\
\hline
\end{tabular}

TNM, tumor node metastasis; SCC, squamous cell carcinoma; HR, hazard ratio; CI, confidence interval.

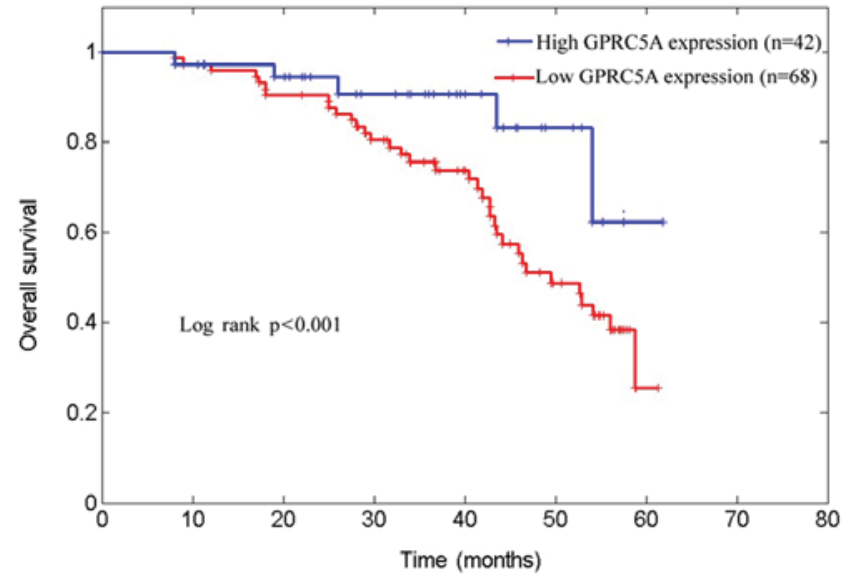

Figure 3. Kaplan-Meier curve analysis of NSCLC patient survival. The 5-year survival rate was $79.2 \%$ in NSCLC patients with high GPRC5A expression. In contrast, the 5 -year survival rate was $26.3 \%$ in NSCLC patients with low GPRC5A expression. The cut-off value for the GPRC5A immunostaining score was set at 6 . NSCLC, non-small cell lung cancer; GPRC5A, G protein-coupled receptor, family $\mathrm{C}$, group 5 member $\mathrm{A}$.

there were no statistically significant differences in GPRC5A expression with respect to age, gender, smoking history, or tumor size (Table I).

We further performed univariate and multivariate analyses. The univariate analysis showed that differentiation, TNM stage, and GPRC5A expression were independent variables; while multivariate analysis revealed that GPRC5A and TNM stage were independent prognostic factors of overall survival for NSCLC patients (Table II).

\section{Discussion}

GPRC5A plays important roles in regulation of the signaling of growth factors and receptors, inflammation, cell death, cell cycle, survival, and cell growth. GPRC5A is expressed in several normal human tissues, and the highest expression levels are observed in the fetal and adult lung (3). Previous studies also have demonstrated that GPRC5A has antitumor activity in NSCLC cells in both animal models and patients $(8-10,14)$. In this study, we assessed GPRC5A expression in normal lung tissues, NSCLC tissues, and paired adjacent noncancerous tissues. In agreement with previous studies $(15,16)$, we observed reduced expression of GPRC5A in NSCLC; although GPRC5A has been reported to be overexpressed in breast, ovarian, and gastric cancers $(17,18)$. Thus, we hypothesized that reduced expression of GPRC5A in NSCLC might indicate that GPRC5A alterations in tumorigenesis and progression vary in different organs and tissues; in addition, in NSCLC, GPRC5A functions as a tumor suppressor gene. Indeed, our current study revealed that reduced GPRC5A expression in NSCLC correlated with poor tumor differentiation and an advanced TNM stage. We also noticed an association between poorly differentiated NSCLC cells and lower GPRC5A expression, confirming the results of previous studies (19-21). Thus, GPRC5A may play important roles in cancer cell differentiation and suppression of NSCLC cell proliferation. Moreover, our Kaplan-Meier analysis results showed that reduced GPRC5A expression was associated with a poor overall survival of NSCLC patients, suggesting that GPRC5A expression may have clinical potential as an independent prognostic indicator for NSCLC patients.

In summary, this retrospective analysis demonstrated that GPRC5A expression was dramatically reduced in NSCLC tissue specimens, compared with normal lung tissues, and that low GPRC5A expression was significantly correlated with poor tumor differentiation and the TNM stage in NSCLC. The data from this study also suggested the clinical impact of GPRC5A as an independent predictor for the overall survival of NSCLC patients. However, further studies are needed to investigate the detailed mechanisms of how GPRC5A is involved in NSCLC development and metastasis. Furthermore, analysis with more clinical samples is necessary for validation of the potential value of GPRC5A as an independent predictor for NSCLC.

\section{Acknowledgements}

Not applicable.

\section{Funding}

This study was supported in part by grants from the Zhejiang Provincial Health-related Research Projects 
(grant no. 2014KYB189), the Zhejiang Provincial Chinese Medicine-related Research Projects (grant no. 2015ZA133), the Medical and Scientific Research Projects of Hangzhou (grant no. 2016Z02) and the Social Development Project of Hangzhou (grant no. 20160533B10).

\section{Availability of data and materials}

The datasets used and/or analyzed during the current study are available from the corresponding author on reasonable request.

\section{Authors' contributions}

EJ, WW, RJX and SM designed the study. EJ, MF, WW, RFX, $\mathrm{HZ}$ and JY acquired the data (provided animals, acquired and managed patients and provided facilities). EJ, MF, WW, RFX, HZ and JY analyzed and interpreted the data (statistical analysis, biostatistics and computational analysis). EJ, MF, WW, RJX, HZ, JY and RFX wrote, reviewed and/or revised the manuscript. EJ, MF, WW, RFX, HZ, JY, RJX and SM collaborated in providing administrative, technical, or material support (reporting or organizing data and constructing databases). RJX and SM supervised the study.

\section{Ethics approval and consent to participate}

Not applicable.

\section{Patient consent for publication}

Not applicable.

\section{Competing interests}

The authors declare that they have no competing interests.

\section{References}

1. Jemal A, Center MM, DeSantis C and Ward EM: Global patterns of cancer incidence and mortality rates and trends. Cancer Epidemiol Biomarkers Prev 19: 1893-1907, 2010.

2. Chen W, Zheng R, Baade PD, Zhang S, Zeng H, Bray F, Jemal A, $\mathrm{Yu}$ XQ and He J: Cancer statistics in China, 2015. CA Cancer J Clin 66: 115-132, 2016.

3. Cheng Y and Lotan R: Molecular cloning and characterization of a novel retinoic acid-inducible gene that encodes a putative $\mathrm{G}$ protein-coupled receptor. J Biol Chem 273: 35008-35015, 1998.

4. Li S, Huang S and Peng SB: Overexpression of G protein-coupled receptors in cancer cells: Involvement in tumor progression. Int J Oncol 27: 1329-1339, 2005.

5. Robbins MJ, Michalovich D, Hill J, Calver AR, Medhurst AD, Gloger I, Sims M, Middlemiss DN and Pangalos MN: Molecular cloning and characterization of two novel retinoic acid-inducible orphan G-protein-coupled receptors (GPRC5B and GPRC5C). Genomics 67: 8-18, 2000.
6. Nagahata T, Sato T, Tomura A, Onda M, Nishikawa K and Emi M: Identification of RAI3 as a therapeutic target for breast cancer. Endocr Relat Cancer 12: 65-73, 2005.

7. Zhou H and Rigoutsos I: The emerging roles of GPRC5A in diseases. Oncoscience 1: 765-776, 2014.

8. Tao Q, Fujimoto J, Men T, Ye X, Deng J, Lacroix L, Clifford JL, Mao L, Van Pelt CS, Lee JJ, et al: Identification of the retinoic acid-inducible Gprc5a as a new lung tumor suppressor gene. J Natl Cancer Inst 99: 1668-1682, 2007.

9. Fujimoto J, Kadara H, Men T, van Pelt C, Lotan D and Lotan R: Comparative functional genomics analysis of NNK tobacco-carcinogen induced lung adenocarcinoma development in Gprc5a-knockout mice. PLoS One 5: e11847, 2010.

10. Barta P, Van Pelt C, Men T, Dickey BF, Lotan R and Moghaddam SJ: Enhancement of lung tumorigenesis in a Gprc5a Knockout mouse by chronic extrinsic airway inflammation. Mol Cancer 11: 4, 2012.

11. Zhong S, Yin H, Liao Y, Yao F, Li Q, Zhang J, Jiao H, Zhao Y, $\mathrm{Xu}$ D, Liu S, et al: Lung tumor suppressor GPRC5A binds EGFR and restrains its effector signaling. Cancer Res 75: 1801-1814, 2015.

12. Detterbeck FC, Boffa DJ and Tanoue LT: The new lung cancer staging system. Chest 136: 260-271, 2009.

13. Livak KJ and Schmittgen TD: Analysis of relative gene expression data using real-time quantitative PCR and the 2(-Delta Delta C(T)) method. Methods 25: 402-408, 2001.

14. Chen Y, Deng J, Fujimoto J, Kadara H, Men T, Lotan D and Lotan R: Gprc5a deletion enhances the transformed phenotype in normal and malignant lung epithelial cells by eliciting persistent Stat 3 signaling induced by autocrine leukemia inhibitory factor. Cancer Res 70: 8917-8926, 2010.

15. Kadara H, Fujimoto J, Men T, Ye X, Lotan D, Lee JS and Lotan R: A Gprc5a tumor suppressor loss of expression signature is conserved, prevalent, and associated with survival in human lung adenocarcinomas. Neoplasia 12: 499-505, 2010.

16. Fujimoto J, Kadara H, Garcia MM, Kabbout M, Behrens C, Liu DD, Lee JJ, Solis LM, Kim ES, Kalhor N, et al: G-protein coupled receptor family C, group 5 , member A (GPRC5A) expression is decreased in the adjacent field and normal bronchial epithelia of patients with chronic obstructive pulmonary disease and non-small-cell lung cancer. J Thorac Oncol 7: 1747-1754, 2012.

17. Jorissen H, Bektas N, Dahl E, Hartmann A, ten Haaf A, Di Fiore S, Kiefer H, Thess A, Barth S and Klockenbring T: Production and characterisation of monoclonal antibodies against RAI3 and its expression in human breast cancer. BMC Cancer 9: 200, 2009.

18. Cheng L, Yang S, Yang Y, Zhang W, Xiao H, Gao H, Deng X and Zhang Q: Global gene expression and functional network analysis of gastric cancer identify extended pathway maps and GPRC5A as a potential biomarker. Cancer Lett 326: 105-113, 2012.

19. Tao Q, Cheng Y, Clifford J and Lotan R: Characterization of the murine orphan G-protein-coupled receptor gene Rai3 and its regulation by retinoic acid. Genomics 83: 270-280, 2004.

20. Ye X and Lotan R: Potential misinterpretation of data on differential gene expression in normal and malignant cells in vitro. Brief Funct Genomic Proteomic 7: 322-326, 2008.

21. Ye X, Tao Q, Wang Y, Cheng Y and Lotan R: Mechanisms underlying the induction of the putative human tumor suppressor GPRC5A by retinoic acid. Cancer Biol Ther 8: 951-962, 2009. 